\title{
Paraffinoma of the Penis: A Case Report
}

\section{Bajaj SK ${ }^{1 *}$, Schakaki S ${ }^{2}$ and van Ahlen $\mathrm{H}^{2}$ \\ ${ }^{1}$ Department of Radiology, Klinikum Osnabrueck, Germany \\ ${ }^{2}$ Department of Urology, Klinikum Osnabrueck, Germany}

*Corresponding author: Dr Satish Bajaj, Consultant Radiologist, Department of Radiology, Klinikum Osnabrueck, Am Finkenhuegel 149076, Osnabrueck, Germany, Email: Satish.bajaj@

\section{Case Report \\ Volume 4 Issue 3}

Received Date: August 10, 2020

Published Date: August 27, 2020

DOI: $10.23880 /$ crij-16000175

Klinikum-os.de

\section{Abstract}

We report on a well-documented case of Paraffinoma of the penis that presented as a urological emergency to our ER due to swelling and necrotic changes of the penis following multiple subcutaneous injections of paraffin oil for the purpose of penis augmentation. The MRI characteristics and its clinical relevance are discussed.

Keywords: Paraffinoma; Sclerosing Lipogranuloma; Magnet Resonance Imaging(MRI)

\section{Introduction}

Penile paraffinoma (synonym: sclerosing lipogranuloma) is an uncommon condition resulting from the application of repeated paraffin injections beneath the skin of the shaft of the penis for the purpose of augmentation of the penis [1]. This kind of procedure is usually performed by nonmedical personnel without technical knowledge and often under unacceptable medical and unhygienic conditions. It may lead to several early complications such as infections, allergic reactions, paraphimosis and pain, whereas paraffinoma is usually a late complication.

\section{Case Report}

A 45 years old patient of Russian origin presented to our emergency department due to diffuse swelling of the penis and severe unbearable pain. He informed us about series of paraffin injections of the penis for the purpose of augmentation over the last 2-3 years.

Inspection of the penis showed discoloration of the skin with gangrenous and ulcerative changes. On palpation, the penis was very tender and showed a diffuse induration of the penile shaft. Multiple lymph-nodes were palpable in the inguinal region on both sides. No urethral discharge was seen. There was no history of venereal diseases. Patient was HIV negative.
CT scan of the abdomen showed swelling, oedema and diffuse enhancement of the subcutaneous tissue without delineations of an abscess. No abnormality of the corpora cavernous of the penis was observed. Multiple reactive inguinal lymph nodes were seen (Figure 1). Urethrogram was performed without detection of any abnormality (not demonstrated).

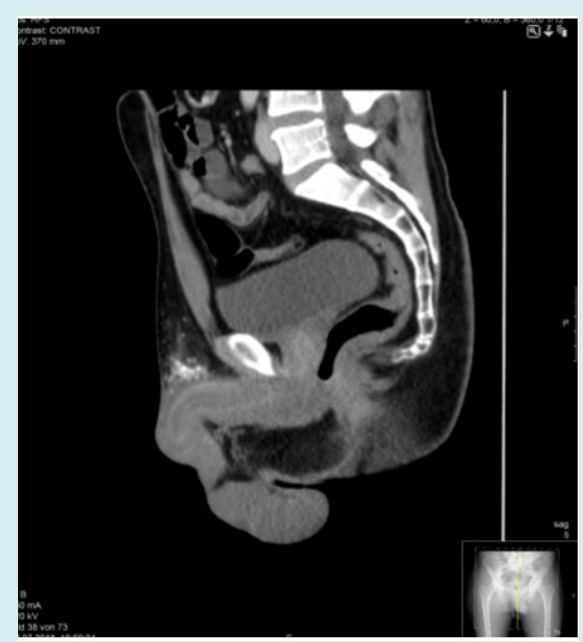

Figure 1: CT scan with contrast media, sagittal view showing diffuse swelling of the penile schaft without macroabscesses. Periglandular hyperdensities (arrow), probably calcified paraffin substrates. 
MRI in the T1 and T2 Sequences showed a diffuse swelling of the shaft with appreciable paraffin substrates (Figure 2) whereas contrast enhanced sequences delineated micro-abscesses on the dorsal aspect of the penis with profuse inflammatory changes of the surrounding areas (Figure 3).

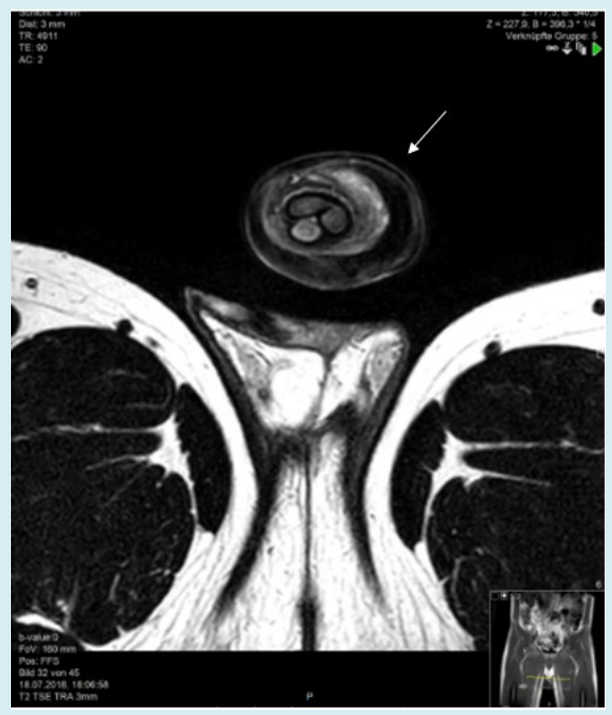

(a)

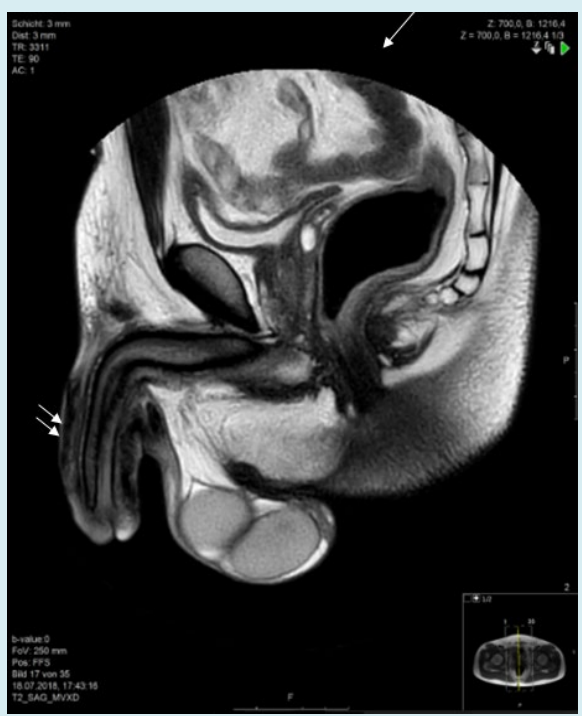

(b)

Figure 2: $(\mathrm{a}, \mathrm{b})$ : T2 w TSE MRI sequence axial and sagittal showing diffuse swelling (arrow) of the penis. No obvious delineation of the abscesses. Testes appear normal on both sides. The calcified paraffin substrates appear as signal hypointensities (double arrow).

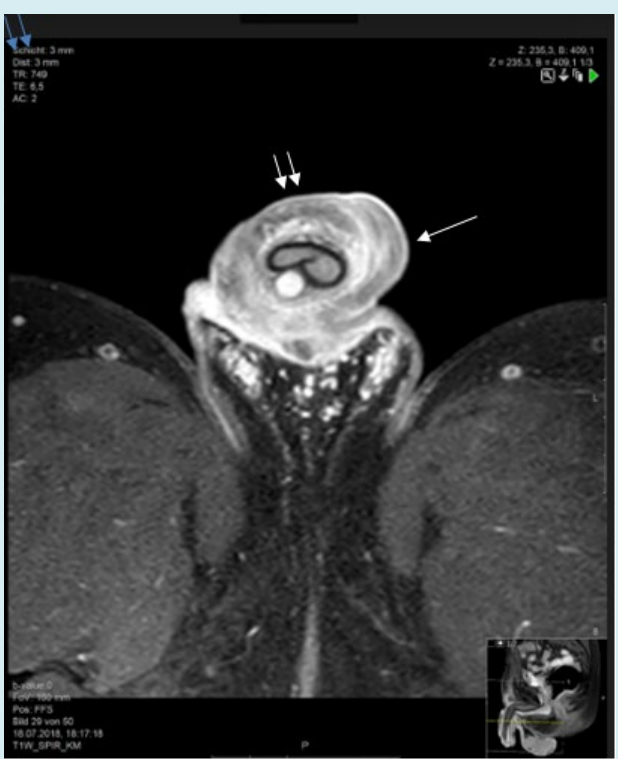

(a)

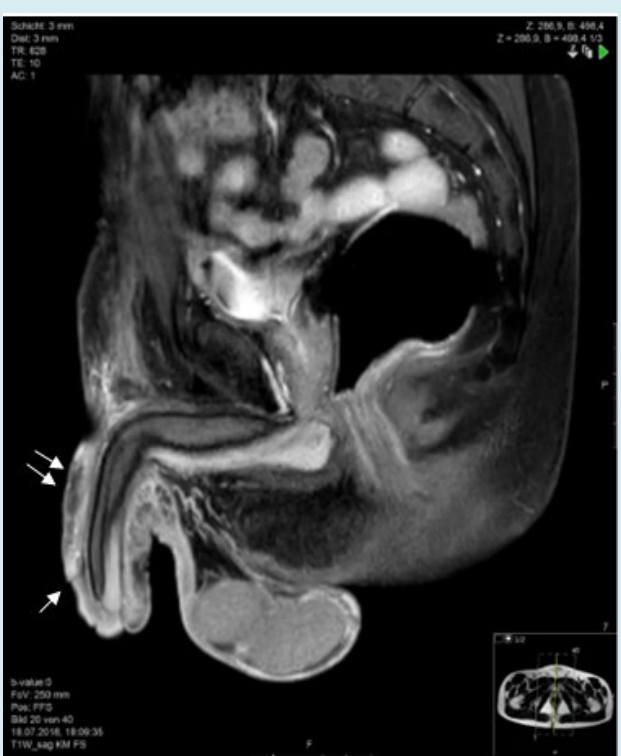

(b)

Figure 3: (a,b): T1 w SE axial and sagittal with Fat-suppression and contrast enhanced sequence demonstrated diffused enhancement of the peri-cavernosal soft tissue of the penis with small delineated non-enhancing areas probably microabscesses. Increased vascularity (arrow) around the penis as sign of profuse inflammatory.

On the basis of the history, as well as clinical and radiological findings, diagnosis of Paraffinoma of the penis was made. The patient was admitted for surgical treatment. Complete debridement of the subcutaneous tissue was 


\section{Clinical Radiology \& Imaging Journal}

carried out by opening up to the level of Buck's fascia of the penis with removal of micro abscesses and inflamed, granulomatous tissue. Multiple drainages were placed and routine surgical dressing was done. The histology of the resected tissue showed extensive foreign body reaction, most probably corresponding to the paraffin injections. No evidence of malignancy or any other granulomatous conditions was found. Bacteriology demonstrated superinfection from Enterobacter cloacae and Staphylococcus aureus which was treated with a combination of Ampicillin/Sublactam and Metronidazole with a good clinical response. The patient was discharged after 10 days and was advised follow up in the outpatient department for the eventual repair of the skin of the penis (Graft).

\section{Discussion}

Penile augmentation for visual improvement of penis size and girth sexual pleasure is not an unusual practice among low social economic groups, prisoners, soldiers and sex-workers, and is relatively more prevalent in the east compared to the western world.

Augmentation by implanting solid particles is known as Penis marble (Fiji), fang muk (Thailand), chagan balls (Korea), bullets (Philippines) and tancho balls (Japan) [2,3].

Injection of high viscosity inert liquid material like paraffin oil, mineral oil and petroleum jelly had been also widely practised in the early $20^{\text {th }}$ century, but has been since abandoned.

Penile paraffinoma is apparently an uncommon urological emergency condition in the western part of the Europe [4-6].

Paraffin is a solid substance, but when heated becomes semisolid and on cooling solidifies again. Due to this principle, it has been widely used in the past for cosmetic surgeries to repair palatal defects, in hernia repair, urinary fistulas, for penile reconstruction and augmentations and removal of facial wrinkles. Robert Gersuny, an Austrian surgeon in 1899 reportedly used mineral oil injection in urological cases to substitute testes in patients who underwent orchiectomy [7]. This technique had been used for breast augmentation in the early $20^{\text {th }}$ century as well.

The human body has no enzyme or mechanism to remove interstitial foreign substrates like mineral oils from the body [6]. Hence when such substances are injected in any part of the body, they are deposited permanently at the injected sites due to a granulomatous reaction.

Due to severe complications of the paraffin injections, it has been abandoned in developed countries but is still in practice in underdeveloped countries as means of easy, low cost fulfilment of demands for penile enlargement.

Early complications of paraffin injections are infections, allergy whereas long term limitations are paraphimosis, constant tenderness and pain, pain on erection and ejaculation as well as gangrenous changes due to the condition called sclerosing lipogranuloma [7-9].

Other causes of subcutaneous penile pathologies should always be considered in the differential diagnosis. Implantation of beads (Glass, stones, bullets, grains of rice, pearls, and jewels) under the skin of the penis to enhance coital excitement and orgasm is widely prevalent in the Far East and south East Asia, particularly in Thailand [9-12]. Glass nodules are called Tacho's nodules. Silicone granuloma is another cause of artificial penile nodules. Allergic contact dermatitis, ossifications of the penis as an extreme variants pf Peyronie "s disease, subcutaneous angiitis of the penis may be other causes of non-infectious, nonneoplastic subcutaneous nodules.

A history of paraffin injection is a lead for a probable diagnosis, although this is often denied by the patients. Presence of paraffin in histological specimen confirms the diagnosis. Ultrasound is a good tool in initial stages to localize the beads or oil, but will not be easy to perform in acute stages due to tenderness and pain. CT scan has a limited role in this condition because of the risk of radiation to genital organs particularly in young patients and due to poor tissue characterisations. New generation CT scanners with spectral techniques can be very useful in differentiating the foreign bodies from normal tissues and the radiation exposure can be substantially reduced. MRI is the modality of choice due to better tissue characterisation without any radiation risks. It can be frequently repeated in follow-ups.

Paraffinomas are managed by complete removal of all the granulomatous tissue containing paraffin to avoid relapse. It is essential not to damage the neurovascular bundle and the Buck's fascia. A single or multiphasic skin-repair with different flap techniques may be necessary once the healing process is complete [12-15].

In conclusion, penile nodules may be a manifestation of diverse pathological entities including infectious, inflammatory and neoplastic process. Although Paraffinoma is an uncommon condition, radiologists should be familiar with it in order to avoid erroneous diagnosis. Awareness of the availability of minimal-invasive, low cost and safe techniques for penis enlargement in the public can contribute towards prevention of complications as seen in this case. 


\section{References}

1. Bjurlin MA, Carlsen J, grevious M, Jordan MD, Taylor A, et al. (2010) Minieral oil-induced sclerosing lipograloma of the penis. J Clin Aesthet Dermatol 3(9): 41-44.

2. Glimore WA, Weigand DA, Burgdorf WHC (1983) Penile nodules in Southeast Asian men. Arch Dermatolo 119(5): 446-447.

3. Levy G. Mercer D, Amosi D, Arad E (2009) Self implanted artificial nodules a computed tomographiy mimic of penile pathology. Urogenital Radiology 49(2): 236-238.

4. Cohen JL, Keoleian CM, Krull EA (2001) Penile paraffinoma; self injection with mineral oil. Journal American Academy Dermatology 45(6): 251-253.

5. Santos A, Chaveiro G, Nunes J, Fonseca J, Cardosaj (2003) Penile paraffinoma. Journal of the European academy of Dermatology 17(5): 583-584.

6. Picozzi, SC, Carmignani L (2010) Paraffnoma of the penis. International Journal Emergency Medicine 3(4): 557-558.

7. Gersuny R (1903) Harte und weiche raraffinprothesen. Zentralbl Chir 30: 1.

\section{Clinical Radiology \& Imaging Journal}

8. Steffens J, Kosharskyy R, Heibl B, Schonberger P, Rottger $P$, et al. (2000) Paraffinoma of the external genitalia after autoinjection of Vaseline. European Urology 38(6): 778781.

9. Bayraktar N, Nasar I (2012) Case reports in Urology.

10. Nitidahaprabhas $P$ (1975) Artificial nodules: case reports from Thailand. Br J Urol 47(40): 463.

11. Sundaravej K, Suchato C (1974) Tancho's nodules. Australas radiol 18(4): 453-454.

12. Murao GB, Dami A, Farina U (1996) Paraffinoma of the penis: one stage repair. Arch Esp Urol 49(6): 648-650.

13. Kim SW, Yoon BI, Ha US, Kim SW, Cho YH, et al. (2014) Treatment of paraffin induced lipogranuloma of the penis by bipedicled scrotal flap with Y-V incision. Ann plast Surg 73(6): 692-695.

14. Shin YS, Zhao C, Park JK (2013) New reconstruction surgery for penile paraffinoma to prevent necrosis of ventral skin. Urology 81(2): 437-441.

15. Dellis AE, Nastos K, Panagiotis TA (2017) Minimal surgical management of penile paraffinoma after subcutaneous penile paraffin injection. Arab J Urol 15(4): 387-390. 